\title{
Transcranial direct-current stimulation protects against cerebral ischemia-reperfusion injury through regulating Cezanne-dependent signaling
}

Jing Cheng

Wuhan University

Yan-Qin Fan

Wuhan University

Hong-Xiang Jiang

Wuhan University

Song-Feng Chen

Wuhan University

\section{Juan Chen}

Huazhong University of Science and Technology - Main Campus: Huazhong University of Science and

Technology

Xin-Yu Liao

Kunming Medical University

Ying-Ying Zou

Kunming Medical University

Hai-yang Lan

Army Medical University

Yu Cui

Qingdao University

Zhi-Biao Chen

Wuhan University

Qianxue Chen ( $\sim$ chenqx666@whu.edu.cn )

Wuhan University https://orcid.org/0000-0002-9413-1030

Qi Wan

Qingdao University

\section{Research Article}

Keywords: Transcranial direct-current stimulation, ischemic stroke, cerebral ischemia-reperfusion injury, Cezanne, SIRT6, DNA DSB 
Posted Date: April 12th, 2021

DOl: https://doi.org/10.21203/rs.3.rs-389968/v1

License: (c) (i) This work is licensed under a Creative Commons Attribution 4.0 International License. Read Full License

Version of Record: A version of this preprint was published at Experimental Neurology on November 1st, 2021. See the published version at https://doi.org/10.1016/j.expneurol.2021.113818. 


\section{Abstract}

Transcranial direct-current stimulation (tDCS) is proved safe and shows therapeutic effect in cerebral ischemic stroke in clinical trials. But the underlying molecular mechanisms remain unclear. Here we show that tDCS treatment reduces the infarct volume after rat cerebral ischemia-reperfusion (I/R) injury and results in functional improvement of stroke animals. At the cellular and molecular level, tDCS suppresses I/R-induced upregulation of Cezanne in the ischemic neurons. Cezanne inhibition confers neuroprotection after rat I/R and oxygen glucose deprivation (OGD) in the cortical neuronal cultures. Inhibiting Cezanne increases the level of SIRT6 that is downregulated in the ischemic neurons. Suppressing SIRT6 blocks Cezanne inhibition-induced neuroprotective effect and overexpressing SIRT6 attenuates OGD-induced neuronal death. We further show that downregulating Cezanne reduces DNA double-strand break (DSB) through upregulation of SIRT6 in OGD-insulted neurons. Together, this study suggests that Cezannedependent SIRT6-DNA DSB signaling pathway may mediate the neuroprotective effect of tDCS in ischemic neurons.

\section{Introduction}

Stroke is the leading cause of death and long-term disability [1]. Ischemic stroke is caused by arterial embolism, resulting in cerebral hypoxia and glucose deficiency. Timely blood reperfusion is essential for reducing brain cell damage, however, blood supply recovery after a period of ischemia leads to ischemiareperfusion (I/R) injury [2].

Transcranial direct-current stimulation (tDCS) is a noninvasive brain stimulation and has been proven safe and shown neuroprotective effect for stroke patients in clinical trials [3-5]. Experimental studies have shown that tDCS significantly protects against ischemic neuronal death and reduces the neurological impairment in rat middle cerebral artery occlusion (MCAO) [6,7]. However, the cellular and molecular mechanisms mediating the neuroprotective effect of $t D C S$ remain unclear.

Cezanne is a deubiquitylase that is a member of the ovarian tumor protease family [8]. Cezanne lyses ubiquitin bonds from lys11-linked diubiquitin chains and to participate in a variety of cellular functions regulated by ubiquitin [9]. Current studies have shown that Cezanne can regulate cell cycle, promote tumor growth, and inhibit NF-KB pathway-mediated inflammatory response $[10,11]$. While studies have been focused on the role of Cezanne in tumorigenesis [12,13], little is known about the role of Cezanne in cerebral ischemic injury.

SIRT6 is a member of Sirtuins family which is specifically localized in the nucleus. The Sirtuins family is the nicotinamide adenine dinucleotide (NAD) ${ }^{+}$-dependent third class of histone deacetylases (HDACs). The family members regulate many biological processes including cellular metabolism, DNA repair, and cell survival [14]. SIRT6 has been reported to promote resistance to DNA damage, oxidative stress, and inhibit genomic instability in metabolic homeostasis [15]. SIRT6 can reduce I/R-induced myocardial and 
hepatic injury [16,17]. SIRT6 was highly expressed in the brain [18] and may play a protective role in cerebral I/R [19].

DNA damage is involved in cell death in the pathogenesis of $\mathrm{I} / \mathrm{R}$ injury and occurs in the early stage of reperfusion [20]. With the extension of reperfusion time, DNA damage is aggravated and DNA doublestrand break (DSB) occurs [21]. Non-homologous terminal junction and homologous recombination are two main approaches to repair DNA DSB [22]. Ku70 is a DNA repair protein which could bind to DNA breaks, repair DNA DSB, and trigger DNA repair pathways [23]. Study suggests that the higher the protein level in Ku70, the more the DNA DSB is repaired [24].

In this study, we investigated the role of Cezanne-SIRT6-DNA DSB-p53 signaling pathway in I/R-induced rat ischemic brain injury. We show that tDCS protects against ischemic neuronal injury through regulating the Cezanne-dependent signaling.

\section{Results}

\section{tDCS is neuroprotective in rat cerebral I/R injury}

To verify the neuroprotective role of tDCS, we tested the effect of tDCS in the rat MCAO model. We show that tDCS promotes functional recovery after I/R (Fig. 1A-2C). Compared with rats in the Control group, rats treated by tDCS scores lower in mNSS test and Beam-walking test, and higher in MST test on day 3, 7 and 14 (Fig. 1A-2C). In agreement with the neurobehavioral tests, 2,3,5-triphenyltetrazolium chloride (TTC) staining data show that tDCS treatment decreases the infarct volume at $24 \mathrm{~h}$ after MCAO (Fig. 1D). These results confirm that tDCS is neuroprotective after rat cerebral I/R in our experimental conditions.

\section{tDCS suppresses I/R-induced increase of Cezanne in the ischemic neurons}

We found that the level of Cezanne is increased in ischemic neurons after cerebral I/R injury (Fig. 2A-B). The increase of Cezanne is also detected in the injured neurons of cultured primary neurons subjected to OGD (Fig. 2C). Interestingly, we show that tDCS treatment blocks the increase of Cezanne in the ischemic brain tissues after rat MCAO (Fig. 2D). In consistent with the in vivo results, direct-current stimulation (DCS) suppresses the increase of Cezanne in the injured neurons in the cortical cultures subjected to OGD (Fig. 2E). Together, these results suggest that tDCS downregulates I/R-induced increase of Cezanne in the ischemic neurons.

\section{Downregulating Cezanne protects against OGD-induced neuronal death}

The observed increase of Cezanne and the downregulation of Cezanne by tDCS in the ischemic neurons lead us to test whether Cezanne exerts its effect on neuronal survival in OGD-insulted neurons. While lentiviral shRNA control has no effect on OGD-induced neuronal death in cultured cortical neurons, lentiviral Cezanne siRNA treatment increases neuronal viability and reduces LDH release (Fig. 3A-B). These results indicate that downregulating Cezanne is neuroprotective in OGD-induced neuronal injury. 


\section{SIRT6 is a downstream signal of Cezanne in ischemic neurons}

To determine how the increase of Cezanne promotes ischemic neuronal death, we set up to investigate the downstream signaling mediating the effect of Cezanne in the ischemic neurons. We first show that SIRT6 is decreased in the injured neurons after cerebral ischemia-reperfusion injury (Fig. 4A-B).

Consistent with in vivo results, a reduced expression of SIRT 6 are also observed in cultured neurons after OGD insult (Fig. 4C). We then found that knockdown of Cezanne by lentiviral shRNA blocks the injuryinduced reduction of SIRT6 after both rat cerebral I/R injury and OGD insult (Fig. 5A-B). Moreover, we show that SIRT6 overexpression by transfection of cDNA-SIRT6 in cultured cortical neurons does not alter the expression of Cezanne (Supplementary Fig. 1), supporting SIRT6 as a downstream signal of Cezanne.

\section{SIRT6 mediates the effect of Cezanne on cell survival in OGD-insulted neurons}

To verify whether SIRT 6 mediates the effect of Cezanne on neuronal survival after $\mathrm{I} / \mathrm{R}$, cultured neurons were transfected with lentiviral cDNA-SIRT6 or SIRT6 shRNA before OGD insult. We show that SIRT6 overexpression ameliorates OGD-induced neuronal injury (Fig. 6A-B), and that the knockdown of SIRT6 increases OGD-induced neuronal death. In neurons transfected with lentiviral SIRT6 shRNA and/or Cezanne shRNA, however, the knockdown of SIRT6 attenuates the knockdown of Cezanne-induced neuroprotection in the OGD-insulted neurons (Fig. 6C-D). Together, these data suggest that SIRT6 acts downstream to mediate the effect of Cezanne on the survival of ischemic neurons.

\section{SIRT6 mediates the regulation of DNA DSB by Cezanne in OGD-insulted neurons}

As Ku70 is a marker of DNA DSB, we tested the level of Ku70 in OGD-insulted neurons. We first show that knockdown of Cezanne by lentiviral Cezanne shRNA increases the level of Ku70 in OGD-insulted neurons. We then treated the cortical cultures with lentiviral SIRT 6 shRNA and Cezanne shRNA and we found that the knockdown of SIRT6 attenuates the knockdown of Cezanne-induced increase of Ku70 in the OGDinsulted neurons (Fig. 7A). Moreover, we show that SIRT6 overexpression by lentiviral cDNA-SIRT6 transfection upregulates the levels of Ku70 (Fig. 7B). These data indicate that Cezanne exerts its effect through SIRT6-DNA DSB signaling pathway in OGD-insulted neurons.

\section{Discussion}

Increasing evidence suggests that tDCS is a potential therapy for ischemic stroke [3-7]. In this study, we show that tDCS treatment reduces the infarct volume after rat I/R injury and results in functional improvement of stroke animals. To understand the molecular mechanism underlying the role of tDCS in cerebral I/R injury, we investigated how intracellular signaling mediates the neuroprotective effect of tDCS in rat model of ischemic stroke. Our results provide evidence suggesting that Cezanne downregulation by tDCS may exert its neuroprotective effect through SIRT6-DNA DSB signaling pathway.

Cezanne is a deubiquitylase that stabilizes target proteins by hydrolyzing ubiquitin chains $[25,26]$. Cezanne can regulate the cell cycle, promote cell growth, and inhibit NF-KB-mediated inflammatory 
response $[10,11]$. Our data for the first time demonstrate that Cezanne is increased in the ischemic neurons after rat cerebral I/R injury. Downregulating Cezanne protects against OGD-induced neuronal death. Interestingly, we found that tDCS suppresses the increase of Cezanne in the ischemic neurons. These findings suggest that tDCS confers neuroprotection through suppression of Cezanne in the ischemic neurons.

To reveal how Cezanne exerts its effect, we uncover SIRT6 as the downstream signal that mediates the role of Cezanne in the ischemic neurons. SIRT6 is a member of the sirtuins family, which is known to regulate various non-histone proteins associated with brain function, including circadian rhythm, neurogenesis, synapses, cognition and myelin formation [27,28]. Recent studies also show that the sirtuins family is involved in ischemic brain injury $[18,29]$. In the ischemic neurons, SIRT 6 overexpression reduced oxidative stress by activating NRF2 and its target genes HO-1 and SOD [30]. SIRT6 inhibits the release of high mobility group box 1 (HMGB1), a known mediator of cerebral ischemic inflammation, in neuroblastoma cells (SH-SY5Y) after OGD insult [18]. In this study, we show that SIRT6 is decreased in the injured neurons after rat cerebral ischemia-reperfusion injury as well as in the cultured neurons after OGD insult. Importantly, we found that knockdown of Cezanne blocks the injury-induced reduction of SIRT6 after both rat cerebral I/R injury and OGD insult. SIRT6 overexpression in cultured cortical neurons does not alter the expression of Cezanne. In addition, we show that SIRT6 overexpression ameliorates OGD-induced neuronal injury, and that the knockdown of SIRT6 increases OGD-induced neuronal death. Moreover, we found that the knockdown of SIRT6 attenuates the knockdown of Cezanne-induced neuroprotection in the OGD-insulted neurons. Together, these results lead us to conclude that SIRT 6 acts downstream to mediate the effect of Cezanne on the survival of ischemic neurons.

It is unclear how SIRT6 is regulated by Cezanne. It is possible that the transcription factor E2F1 may mediate the regulation of SIRT 6 by Cezanne since Cezanne inhibition is shown to promote the deubiquitination of E2F1. In addition, it has been reported that E2F1 suppression reduces the direct binding of E2F1 to the SIRT6 promoter, and ultimately promotes SIRT6 expression [31, 32]. Future studies will be performed to test this possibility.

It has been reported that I/R injury-induced DNA damage occurs in the early stage of reperfusion [21]. With the extension of reperfusion time, DNA injury intensifies and it results in DNA DSB and fragmentation [21]. Ku70 is a DNA repair protein that can bind to DNA break ends and trigger DNA repair pathways to reduce DNA DSB [23]. Higher Ku70 level indicates lower DNA DSB level [24]. Recent studies indicate that SIRT 6 reduces DNA DSB and DNA damage by up-regulating the repair pathways of nonhomologous terminal junction and homologous recombination [22]. We found that knockdown of Cezanne increases the level of Ku70 in OGD-insulted neurons. We then found that the knockdown of SIRT6 attenuates the knockdown of Cezanne-induced increase of Ku70 in the OGD-insulted neurons. Moreover, we show that SIRT6 overexpression upregulates the levels of Ku70. These data indicate that SIRT6 may mediate the effect of Cezanne through DNA DSB in the injured neurons. 
Collectively, this study for the first time demonstrates a role of Cezanne in cerebral ischemia injury. Our results suggest that Cezanne-dependent SIRT6-DNA DSB signaling pathway may mediate the neuroprotective effect of tDCS in ischemic neurons (Supplementary Fig. 2).

\section{Materials And Methods}

\section{Animals}

In a temperature control room $\left(23-25^{\circ} \mathrm{C}\right)$, adult male Sprague-Dawley (SD) rats were housed in three rats per cage at a $12 \mathrm{~h} \mathrm{light/dark}$ cycle, weighing 230 to $250 \mathrm{~g}$, free access to drinking water and food. Adapt to the environment at least 3 days before the experiment. All animal use and experimental protocols were approved and carried out in compliance with the IACUC guidelines and the Animal Care and Ethics Committee of Wuhan University School of Medicine and Qingdao University School of Medicine. Randomization was used to assign samples to the experimental groups, and to collect and process data. The experiments were performed by investigators blinded to the groups for which each animal was assigned.

\section{Local cerebral ischemia and infarct size measurement}

Transient cerebral ischemia induced by suture occlusion technique [33]. Male SD rats in each group were randomly sampled to select rats with the same weight range for experiment. Male SD rats were anesthetized with a mask of $4 \%$ isoflurane in $70 \% \mathrm{~N}_{2} \mathrm{O}$ and $30 \% \mathrm{O}_{2}$. The midline neck incision, careful exposure and dissection of the right external carotid artery (ECA), from the ECA insertion line into the right internal carotid artery occlusion of the right middle cerebral artery (MCA) (about $22 \mathrm{~mm}$ ). After occlusion for 90 minutes, the wire plug was removed to allow reperfusion, the ECA was ligated, and the wound was closed. Sham-operated rats underwent the same surgery and/or intraventricular injection, except that the plug was inserted and immediately withdrawn. The body temperature was maintained at $37.0 \pm 0.5^{\circ} \mathrm{C}$ using a heating pad and a heat lamp. 24 hours after MCAO, after anesthesia with $4 \%$ isoflurane in $70 \%$ $\mathrm{N}_{2} \mathrm{O}$ and $30 \% \mathrm{O}_{2}$, rats were reperfused with ice-cold $0.9 \%$ saline, and then the brain was quickly removed for Western blotting and TTC (2,3,5-triphenyltetrazolium chloride) staining. The rats were sacrificed ( $\mathrm{n}=$ 56) and the brains were removed for Western blotting. Tissues surrounding the infarcts of the ipsilateral hemispheres were homogenized in RIPA buffer for 30 minutes on ice using a tissue mill. Tissue lysates were then centrifuged at $12000 \times \mathrm{g}$ for 15 minutes at $4^{\circ} \mathrm{C}$ and all protein concentrations were determined using a BCA protein assay device. The rats were sacrificed $(n=40)$ and the brain was removed for TTC staining to assess the volume of cerebral infarction [34]. The brain was placed in a cooled matrix and cut into $2 \mathrm{~mm}$ coronal sections. Each section was placed in a $10 \mathrm{~cm}$ dish and incubated with $2 \%$ TTC in phosphate buffered saline for 30 minutes in an oven at $37^{\circ} \mathrm{C}$. The sections were fixed in $4 \%$ paraformaldehyde in a $4^{\circ} \mathrm{C}$ refrigerator. All image acquisition, processing and analysis in a blind manner and under controlled environmental lighting. Scanned images were analyzed using Image J software and infarct data from all groups were expressed as the ratio of infarcted area to total brain slice area [34]. 


\section{Intraventricular injection (i.c.v)}

The rats were anesthetized with a mixture of $30 \% \mathrm{O}_{2}$ and $70 \% \mathrm{~N}_{2} \mathrm{O}$ with $4 \%$ isoflurane in a sealed fluoroscopy box. When the rats are deeply anesthetized, we will use the ear bars and the upper incisor bar

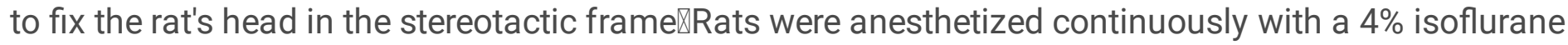
mask. Next, a small sagittal incision was made in the rat's head and the anterior iliac crest was positioned as an anatomical reference point. The ventricles (from the anterior iliac crest: $1.5 \mathrm{~mm}$; anteroposterior, - $0.8 \mathrm{~mm}$; depth, $3.5 \mathrm{~mm}$ ) were connected to a Hamilton microinjector via a polyethylene tube using a 23-gauge needle at a drug infusion rate of $8 \mu \mathrm{l} / \mathrm{min}$ for $2 \mathrm{~min}$. Appropriate needle placement was verified by taking a few microliters of clear cerebrospinal fluid into a Hamilton microsyringe.

\section{Cortical Neuron Culture and OGD Insult}

Cortical neuronal cultures were prepared from SD rats on day 17 of gestation as we described in previous reports. Pregnant rats were anesthetized with $4 \%$ isoflurane in $70 \% \mathrm{~N}_{2} \mathrm{O}$ and $30 \% \mathrm{O}_{2}$ and sacrificed by cervical dislocation. The rats were spray-sterilized with $70 \%$ ethanol and the embryos were removed. Quickly break the embryo and place the cortex in the cold after removing the meninges plating medium \neurobasal medium, 2\% B-27 supplement, 0.5\% FBS, $0.5 \mathrm{mM} \mathrm{L-glutamax}$ and $25 \mathrm{mM}$ glutamic acid囚. Cortical neurons were suspended in a plating medium and plated on a Petri dish coated with poly-Dlysine. Half of the plating medium was taken out and replaced with maintenance medium (Neurobasal medium, 2\% B-27 supplement, and 0.5 mM L-glutamine) in the same manner every 3 days. After 12 days, the cultured neurons were used for experiments [35]. For OGD/R injury, the cells were transferred to a deoxygenated, glucose-free extracellular solution (in mmol/L: $116 \mathrm{NaCl}, 5.4 \mathrm{KCl}, 0.8 \mathrm{MgSO}_{4}, 1.0 \mathrm{NaH}_{2} \mathrm{PO}_{4}$, $1.8 \mathrm{CaCl}_{2}$, and $26 \mathrm{NaHCO}_{3}$ ); into a specialized, humidified chamber and maintained at $37{ }^{\circ} \mathrm{C}, 85 \% \mathrm{~N}_{2} / 10 \%$ $\mathrm{H}_{2} / 5 \% \mathrm{CO}_{2}$ for 60 minutes. The medium was then replaced with fresh maintenance medium containing the appropriate concentration of reagents in a $95 \% \mathrm{O}_{2} / 5 \% \mathrm{CO}_{2}$ incubator for 24 hours during recovery. First transfer the control culture to another extracellular solutionखin mmol/L: $116 \mathrm{NaCl}, 5.4 \mathrm{KCl}, 0.8$ $\mathrm{MgSO}_{4}, 1.0 \mathrm{NaH}_{2} \mathrm{PO}_{4}, 1.8 \mathrm{CaCl}_{2}, 26 \mathrm{NaHCO}_{3}$, and 33 glucose区, and then introduced into the humidified chamber which were maintained at $37^{\circ} \mathrm{C}$ for 60 minutes in $95 \% \mathrm{O}_{2} / 5 \% \mathrm{CO}_{2}$. Then the medium was replaced with fresh maintenance medium for the whole period at $37^{\circ} \mathrm{C}$ in a $95 \% \mathrm{O}_{2} / 5 \% \mathrm{CO}_{2}$ incubator.

\section{tDCS and DCS experimental procedures}

For mice MCAO model, tDCS was applied in mice without re-anesthesia by a constant current stimulator (Schneider Electronics, Gleichen, Germany) that was specifically designed for application of low-intensity currents in small mammals [36]. Epicranial electrode implant was carried out in mice 7 days before the MCAO operation. One electrode was positioned on each side of the cranium in a symmetrical way and fixed with nontoxic glass ionomer cement. The electrode over the ischemic cortex was connected to the cathodal terminal and the other electrode was connected to anodal terminal. Prior to stimulation, epicranial implanted electrode was filled with saline solution. The contact area of the electrode toward the skull was $3.5 \mathrm{~mm}^{2}$. The tDCS was applied in mice at current intensity of $100 \mu \mathrm{A}$ with a current density 
of $2.86 \mathrm{~mA} / \mathrm{cm}^{2}$. Mice underwent tDCS at $3 \mathrm{~h}$ after $\mathrm{l} / \mathrm{R}$ for $10 \mathrm{~min}$, followed by $3 \mathrm{~min}$ rest and then $10 \mathrm{~min}$ stimulation, for a total 8 times of 10 min stimulation. To avoid a stimulation break effect, the current strength was ramped for $10 \mathrm{~s}$. The sham mice underwent the same procedure of stimulated groups, but no current was applied.

For OGD model, steady DCSs at the physiological strength $250 \mathrm{mV} / \mathrm{mm}$ were applied to cultured neurons in culture chambers using methods described previously [37]. For the DCS stimulation, agar-salt bridges were used to connect silver/silver chloride electrodes in beakers of Steinberg's solution, to pools of excess culture medium at either side of the chamber. Field strengths were measured directly at the beginning and end of the observation period. Culture conditions in control were identical except no DCSs were applied. HEPES acid $(20 \mathrm{mM})$ was added to the culture medium, with $\mathrm{pH}$ adjusted to 7.4. The cells were stimulated by DCS at the current strength of $250 \mathrm{mV} / \mathrm{mm}$ for $20 \mathrm{~min}$ at $3 \mathrm{~h}$ after reoxygenation following OGD.

\section{Western Blotting}

Western blotting analysis as described previously [38]. In short, the polyvinylidene difluoride membrane (Millipore, USA) was incubated with primary antibody (Cezanne mouse monoclone antibody, 1:1,000, cat. no. sc-514402; Santa Cruz Biotechnology; SIRT6 rabbit monoclonal antibody, 1:1,000, cat. no. ab191385; Abcam; p53 rabbit polyclonal antibody, 1:1,000, cat. no. \#9282; Cell Signaling Technology; caspase-3 mouse monoclonal antibody, 1:1,000, cat. no. 31A1067; Novus Biologicals; Ku70 and Ku86 mouse

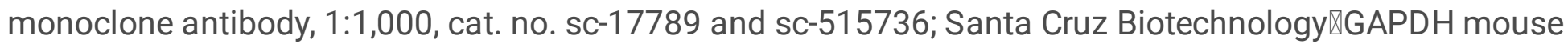
monoclonal antibody, 1: 3,000, cat. no. sc-365062; Santa Cruz Biotechnology) overnight at $4^{\circ} \mathrm{C}$. Primary antibodies were labeled with horseradish peroxidase-conjugated secondary antibody, and protein bands were imaged using SuperSignal West Femto Maximum Sensitivity Substrate (Pierce, USA). The EC3 Imaging System (UVP, USA) was used to obtained blot images directly from the polyvinylidene difluoride membrane. The experimenters were blinded to the groups allocation during the experiment. The quantification of Western blot data was performed using ImageJ software.

\section{Immunofluorescence assay}

Rats were treated with an over dose of isoflurane, then intracardiac perfusion with $0.9 \%$ saline, next put in $4 \%$ paraformaldehyde (PFA) at $4^{\circ} \mathrm{C}$ for $24 \mathrm{~h}$, and followed by transfered into $30 \%$ sucrose solution in 100 $\mathrm{mol} / \mathrm{mL}$ phosphate buffer at $4^{\circ} \mathrm{C}$ for $72 \mathrm{~h}$. Then the brains tissue of rats and human were kept in $4 \%$ paraformaldehyde solution at $4^{\circ} \mathrm{C}$ overnight. Brains tissues were cut into $16 \mu \mathrm{m}$ coronal sections by a Leica VT1000S vibratome (Leica Micro-systems AG, Nussloch, Germany). The brain sections were treated with primary antibody rabbit anti- Cezanne (1:100) from 1:100, Wuhan sanying Biotechnology, China, mouse anti- SIRT6 (1:100) from 1:100, Santa Cruz Biotechnology, USA, rabbit anti- NeuN (neuronalspecific nuclear protein) and mouse anti- NeuN from 1:100, Abcam, USA. The secondary antibody goat anti- Rabbit 488, anti- Mouse 488, goat anti- Rabbit 594,goat anti- Mouse 594 from Molecular Probes 
(Eugene, USA). The sections were photographed by a blinded investigator using an Olympus fluorescent microscope (IX51, Olympus, Japan). Analysed by Image J software (Image J, USA).

\section{Analysis of lactate dehydrogenase release and cell viability}

Lactate dehydrogenase (LDH) release was analyzed using a colorimetric CytoTox 96 Cytotoxicity kit (Promega). Cell viability in the neuronal cultures was evaluated by the ability to take up thiazolyl blue tetrazolium bromide (mingest thiazolyl tetrazolium, MTT) (PowerWave X; BioTek, Winooski, VT). The two methods were performed following the manufacturer's instructions.

\section{Transfection}

shRNA(sh)-Cezanne Lentiviral Particles (cat.no.sc-151945-V; Santa Cruz Biotechnology, Inc.) or Control shRNA Lentiviral Particles (cat.no. sc-108080; Santa Cruz Biotechnology, Inc.) were transfected in primary neurons according to the manufacturer's protocol. Prior to transfection, cells were plated in 6-well or 96well plates and grown to $40-50 \%$ confluence. Cells treated with $10 \mu \mathrm{l} / \mathrm{ml}$ sh-Cezanne or Control shRNA Lentiviral Particles were transfected for $96 \mathrm{~h}$. Select stable clones expressing the shRNA-Cezanne via Puromycin dihydrochloride (cat.no.sc-108071; Santa Cruz Biotechnology, Inc.) selection.

shRNA(sh)-Cezanne Lentiviral Particles (cat.no.sc-151945-V; Santa Cruz Biotechnology, Inc.) or Control shRNA Lentiviral Particles (cat.no. sc-108080; Santa Cruz Biotechnology, Inc.) were transfected according to the manufacturer's protocol. The rats received $16 \mu$ lentiviral supernatant $\left(10^{9}\right.$ infectious units $\left./ \mathrm{ml}\right)$ by intracerebroventricular (i.c.v) injection.

shRNA(sh)-SIRT6 Lentiviral Particles (cat.no.sc-63029-V; Santa Cruz Biotechnology, Inc.) or Control shRNA Lentiviral Particles (cat.no. sc-108080; Santa Cruz Biotechnology, Inc.) were transfected in primary neurons according to the manufacturer's protocol. Prior to transfection, cells were plated in 6-well or 96 -well plates and grown to $40-50 \%$ confluence. Cells treated with $10 \mu \mathrm{l} / \mathrm{ml}$ sh-SIRT6 or Control shRNA Lentiviral Particles were transfected for $96 \mathrm{~h}$. Select stable clones expressing the shRNA-SIRT6 via Puromycin dihydrochloride (cat.no.sc-108071; Santa Cruz Biotechnology, Inc.) selection.

The transfection of the SIRT6 plasmid (Addgene) was conducted using the X-tremeGENE HP DNA Transfection Reagent (Roche) in terms of the manufacturer's instructions. A density of $2 \times 10^{5}$ cells was first seeded in each well of a six-well plate and then transfected with complexes containing $2 \mu \mathrm{g}$ of SIRT6 plasmid or a negative control with pcDNA3.1 and $2 \mu \mathrm{l}$ of the X-tremeGENE transfection reagent. Then, the cells treated with $1 \mu \mathrm{g} / \mu \mathrm{l}$ pcDNA3.1-SIRT6 or negative controlwere incubated under normal condition for $48 \mathrm{~h}$ at $37^{\circ} \mathrm{C}$.

\section{Neurobehavioral Tests}

Total 62 rats were used for neurobehavioral tests. Neurological Severity Scores: The rats were subjected to a modified neurological severity score (mNSS) test as reported previously [39]. These tests are a battery of motor, sensory, reflex, and balance tests, which are similar to the contralateral neglect tests in 
humans. Neurological function was graded on a scale of 0 to 18 (normal score, 0; maximal deficit score, 18).

Beam walk test: The beam walk test measures the animals' complex neuromotor function [40]. The animal was timed as it walked a $(100 \times 2 \mathrm{~cm})$ beam. A box for the animal to feel safe was placed at one end of the beam. A loud noise was created to stimulate the animal to walk toward and into the box [41]. Scoring was based upon the time it took the rat to go into the box. The higher the score, the more severe is the neurological deficit.

Adhesive-removal test: A modified sticky-tape (MST) test was performed to evaluate forelimb function [42]. A sleeve was created using a $3.0 \times 1.0 \mathrm{~cm}$ piece of yellow paper tape and was subsequently wrapped around the forepaw so that the tape attached to itself and allowed the digits to protrude slightly from the sleeve. The typical response is for the rat to vigorously attempt to remove the sleeve by either pulling at the tape with its mouth or brushing the tape with its contralateral paw. The rat was placed in its cage and observed for 30s. Two timers were started: the first ran without interruption and the second was turned on only while the animal attempted to remove the tape sleeve. The ratio of the left (affected)/right (unaffected) forelimb performance was recorded. The contralateral and ipsilateral limbs were tested separately. The test was repeated three times per test day, and the best two scores of the day were averaged. The lower the ratio, the more severe is the neurological deficit.

\section{Statistical Analysis}

Student's $t$ test or ANOVA test was used where appropriate to examine the statistical significance of the differences between groups of data. All results are presented as mean \pm SEM. Significance was placed at $p<0.05$.

\section{Declarations}

\section{Funding statement}

This work was supported by National Key R\&D Program of China (2019YFC0120000; 2018YFC1312300), the National Natural Science Foundation of China (NSFC: 82071385), and the Key Research and Development Project of Shandong (2019JZZY021010) to Q.W. This work was also supported by the Fundamental Research Funds for the Central Universities (2042020kf0090) to Y.F.

\section{Author contributions}

QXC and QW conceived the topic and designed the outline of this review; JC and YQF drafted the manuscript and participated in the cell experiments; HXJ, SFC and JC participated in the design of study and cell experiments; $X Y L, Y Y Z$ and HYL participated in the animal experiments; $Y C$ and ZBC participated in animal model construction and data analysis. All authors whose names appear on the submission approved the version to be published and agree to be accountable for all aspects of the work in ensuring 
that questions related to the accuracy or integrity of any part of the work are appropriately investigated and resolved.

\section{Compliance with ethical standards}

The authors declare no competing financial interests. All animal use and experimental protocols were approved and implemented by the IACUC guidelines and the Animal Care and Ethics Committee of the Wuhan University School of Medicine.

\section{Consent to participate}

$\mathrm{N} / \mathrm{A}$

\section{Consent for Publication}

N/A

\section{Data Availability Statement}

All supporting data are included within the main article. For the original data, please contact the corresponding author.

\section{References}

1. Rathore, S.S., et al., Characterization of incident stroke signs and symptoms: findings from the atherosclerosis risk in communities study. Stroke, 2002. 33(11): p. 2718-21.

2. Yen, T.L., et al., Neuroprotective effects of xanthohumol, a prenylated flavonoid from hops (Humulus lupulus), in ischemic stroke of rats. J Agric Food Chem, 2012. 60(8): p. 1937-44.

3. Hameed, M.Q., et al., Transcranial Magnetic and Direct Current Stimulation in Children. Curr Neurol Neurosci Rep, 2017. 17(2): p. 11.

4. Russo, C., et al., Safety Review of Transcranial Direct Current Stimulation in Stroke. Neuromodulation, 2017. 20(3): p. 215-222.

5. Li, L., et al., Direct-current electrical field guides neuronal stem/progenitor cell migration. Stem Cells, 2008. 26(8): p. 2193-200.

6. Peruzzotti-Jametti, L., et al., Safety and efficacy of transcranial direct current stimulation in acute experimental ischemic stroke. Stroke, 2013. 44(11): p. 3166-74.

7. Zhang, K.Y., et al., Cathodal tDCS exerts neuroprotective effect in rat brain after acute ischemic stroke. BMC Neurosci, 2020. 21(1): p. 21.

8. Evans, P.C., et al., A novel type of deubiquitinating enzyme. J Biol Chem, 2003. 278(25): p. 23180-6.

9. Bremm, A., S.M. Freund, and D. Komander, Lys11-linked ubiquitin chains adopt compact conformations and are preferentially hydrolyzed by the deubiquitinase Cezanne. Nat Struct Mol Biol, 
2010. 17(8): p. 939-47.

10. Bonacci, T., et al., Cezanne/OTUD7B is a cell cycle-regulated deubiquitinase that antagonizes the degradation of $A P C / C$ substrates. EMBO J, 2018. 37(16).

11. Ji, Y., et al., The N-terminal ubiquitin-associated domain of Cezanne is crucial for its function to suppress NF-kappaB pathway. J Cell Biochem, 2018. 119(2): p. 1979-1991.

12. Wang, J.H., et al., Cezanne predicts progression and adjuvant TACE response in hepatocellular carcinoma. Cell Death Dis, 2017. 8(9): p. e3043.

13. Pang, Z., et al., Expressions of insulin-like growth factor receptor-1 and cezanne-1 in lung adenocarcinoma. Med Oncol, 2017. 34(5): p. 78.

14. Matsushima, S. and J. Sadoshima, The role of sirtuins in cardiac disease. Am J Physiol Heart Circ Physiol, 2015. 309(9): p. H1375-89.

15. Mostoslavsky, R., et al., Genomic instability and aging-like phenotype in the absence of mammalian SIRT6. Cell, 2006. 124(2): p. 315-29.

16. Wang, X.X., et al., SIRT6 protects cardiomyocytes against ischemia/reperfusion injury by augmenting FoxO3alpha-dependent antioxidant defense mechanisms. Basic Res Cardiol, 2016. 111(2): p. 13.

17. Zhang, S., et al., SIRT6 protects against hepatic ischemia/reperfusion injury by inhibiting apoptosis and autophagy related cell death. Free Radic Biol Med, 2018. 115: p. 18-30.

18. Lee, O.H., et al., Decreased expression of sirtuin 6 is associated with release of high mobility group box-1 after cerebral ischemia. Biochem Biophys Res Commun, 2013. 438(2): p. 388-94.

19. Cui, X., et al., SIRT6 regulates metabolic homeostasis in skeletal muscle through activation of AMPK. Am J Physiol Endocrinol Metab, 2017. 313(4): p. E493-E505.

20. Wang, S., et al., Cellular NAD replenishment confers marked neuroprotection against ischemic cell death: role of enhanced DNA repair. Stroke, 2008. 39(9): p. 2587-95.

21. Didenko, V.V., et al., Visualization of irreparable ischemic damage in brain by selective labeling of double strand blunt-ended DNA breaks. Mol Med, 2002. 8(12): p. 818-23.

22. Tian, X., et al., SIRT6 Is Responsible for More Efficient DNA Double-Strand Break Repair in Long-Lived Species. Cell, 2019. 177(3): p. 622-638 e22.

23. Featherstone, C. and S.P. Jackson, Ku, a DNA repair protein with multiple cellular functions? Mutat Res, 1999. 434(1): p. 3-15.

24. Kim, G.W., et al., Early decrease in dna repair proteins, Ku70 and Ku86, and subsequent DNA fragmentation after transient focal cerebral ischemia in mice. Stroke, 2001. 32(6): p. 1401-7.

25. Komander, D., M.J. Clague, and S. Urbe, Breaking the chains: structure and function of the deubiquitinases. Nat Rev Mol Cell Biol, 2009. 10(8): p. 550-63.

26. Clague, M.J., et al., Deubiquitylases from genes to organism. Physiol Rev, 2013. 93(3): p. 1289-315.

27. Sosnowska, B., et al., The sirtuin family members SIRT1, SIRT3 and SIRT6: Their role in vascular biology and atherogenesis. Atherosclerosis, 2017. 265: p. 275-282. 
28. O'Callaghan, C. and A. Vassilopoulos, Sirtuins at the crossroads of stemness, aging, and cancer. Aging Cell, 2017. 16(6): p. 1208-1218.

29. Zhang, W., et al., Sirtuin 6 protects the brain from cerebral ischemia/reperfusion injury through NRF2 activation. Neuroscience, 2017. 366: p. 95-104.

30. Kim, S.H., H.F. Lu, and C.C. Alano, Neuronal Sirt3 protects against excitotoxic injury in mouse cortical neuron culture. PLoS One, 2011. 6(3): p. e14731.

31. Moniz, S., et al., Cezanne regulates E2F1-dependent HIF2alpha expression. J Cell Sci, 2015. 128(16): p. 3082-93.

32. Wu, M., E. Seto, and J. Zhang, E2F1 enhances glycolysis through suppressing Sirt6 transcription in cancer cells. Oncotarget, 2015. 6(13): p. 11252-63.

33. Longa, E.Z., et al., Reversible middle cerebral artery occlusion without craniectomy in rats. Stroke, 1989. 20(1): p. 84-91.

34. Wexler, E.J., et al., An objective procedure for ischemic area evaluation of the stroke intraluminal thread model in the mouse and rat. J Neurosci Methods, 2002. 113(1): p. 51-8.

35. Shan, Y., et al., Regulation of PINK1 by NR2B-containing NMDA receptors in ischemic neuronal injury. J Neurochem, 2009. 111(5): p. 1149-60.

36. Liebetanz, D., et al., Safety limits of cathodal transcranial direct current stimulation in rats. Clin Neurophysiol. 2009;120(6):1161-1167.

37. Li, L., et al., Direct-current electrical field guides neuronal stem/progenitor cell migration. Stem Cells. 2008 Aug;26(8):2193-200.

38. Chen, J., et al., A non-ionotropic activity of NMDA receptors contributes to glycine-induced neuroprotection in cerebral ischemia-reperfusion injury. Sci Rep, 2017. 7(1): p. 3575.

39. Chen, J., et al., Intravenous administration of human umbilical cord blood reduces behavioral deficits after stroke in rats. Stroke, 2001. 32(11): p. 2682-8.

40. Clifton, G.L., et al., Marked protection by moderate hypothermia after experimental traumatic brain injury. J Cereb Blood Flow Metab, 1991. 11(1): p. 114-21.

41. Petullo, D., et al., Model development and behavioral assessment of focal cerebral ischemia in rats. Life Sci, 1999. 64(13): p. 1099-108.

42. Sughrue, M.E., et al., An improved test of neurological dysfunction following transient focal cerebral ischemia in rats. J Neurosci Methods, 2006. 151(2): p. 83-9.

\section{Figures}


A

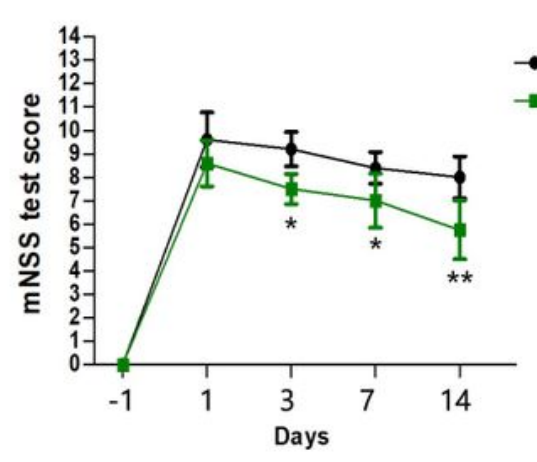

C

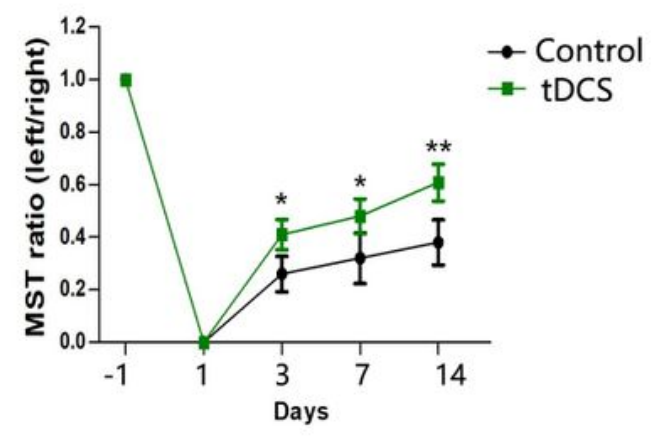

D

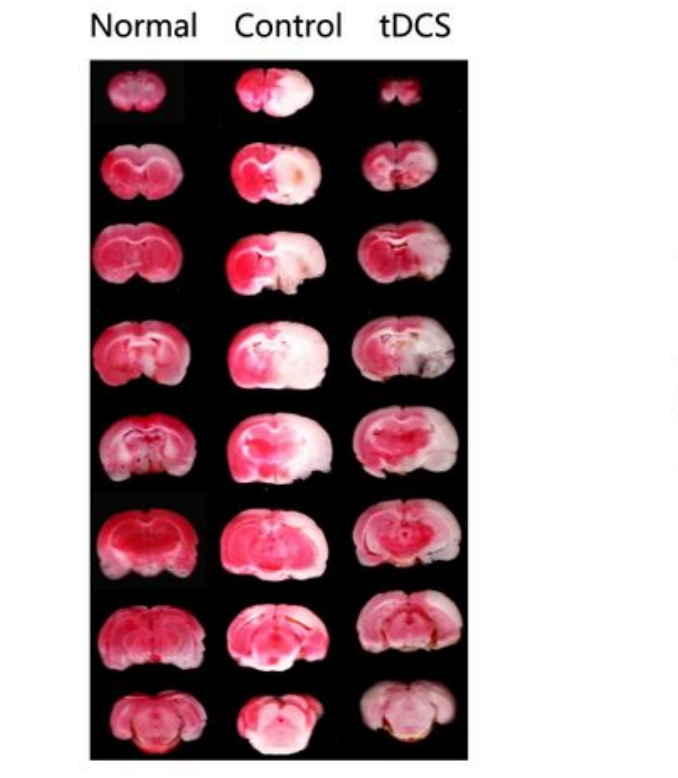

$\rightarrow$ Control

- tDCS

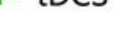

$$
\text { 岕 }
$$

C

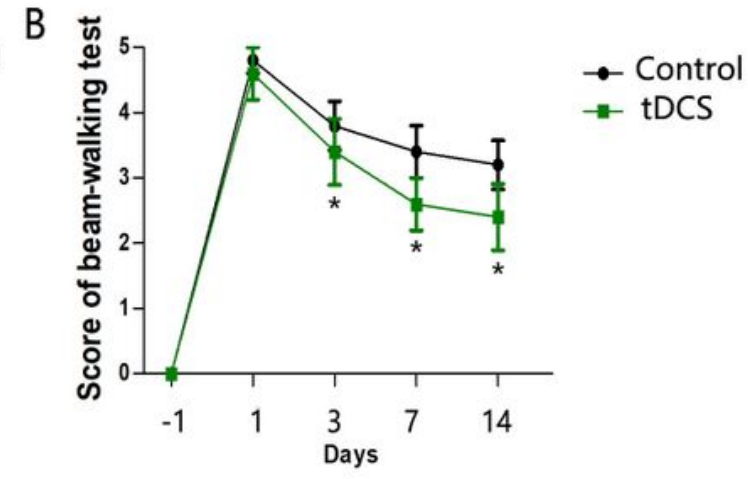

\section{Figure 1}

Neurobehavioral tests and TTC staining in MCAO/R rats with tDCS. (A) Rats with tDCS had lower scores in mNSS test at day 1, 3, 7 and 14 compared to group Control. (B) Rats with tDCS had lower scores in beam-walking test at day 1, 3, 7 and 14 compared to group Control. (C) Rats with tDCS had higher ratio in MST test at day 1, 3, 7 and 14 compared to group Control. (D) TTC staining showed the infarct volume of the rat brain after MCAO/R(90min/24h) in each group. ( $\mathrm{n}=6$ for each group; * $\mathrm{P}<0.05$ or ** $\mathrm{p}<0.01$ 
compared to Control, two-way ANOVA test, followed by Bonferroni post hoc test). Control: rat middle cerebral artery occlusion/reperfusion with nonstimulated tDCS; tDCS: rat middle cerebral artery occlusion/reperfusion with tDCS.

A

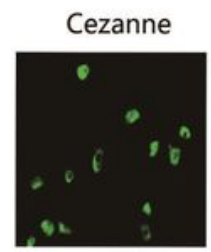

NeuN
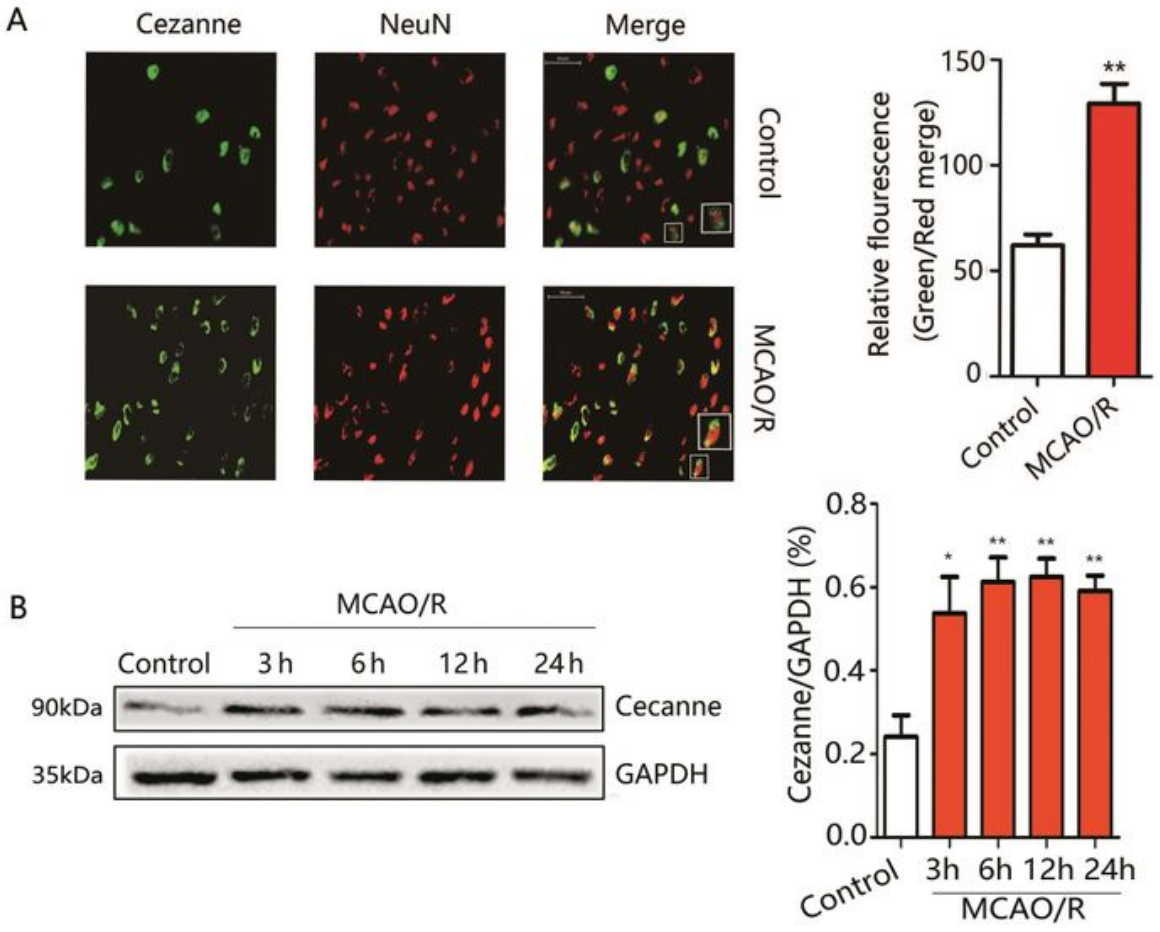

C
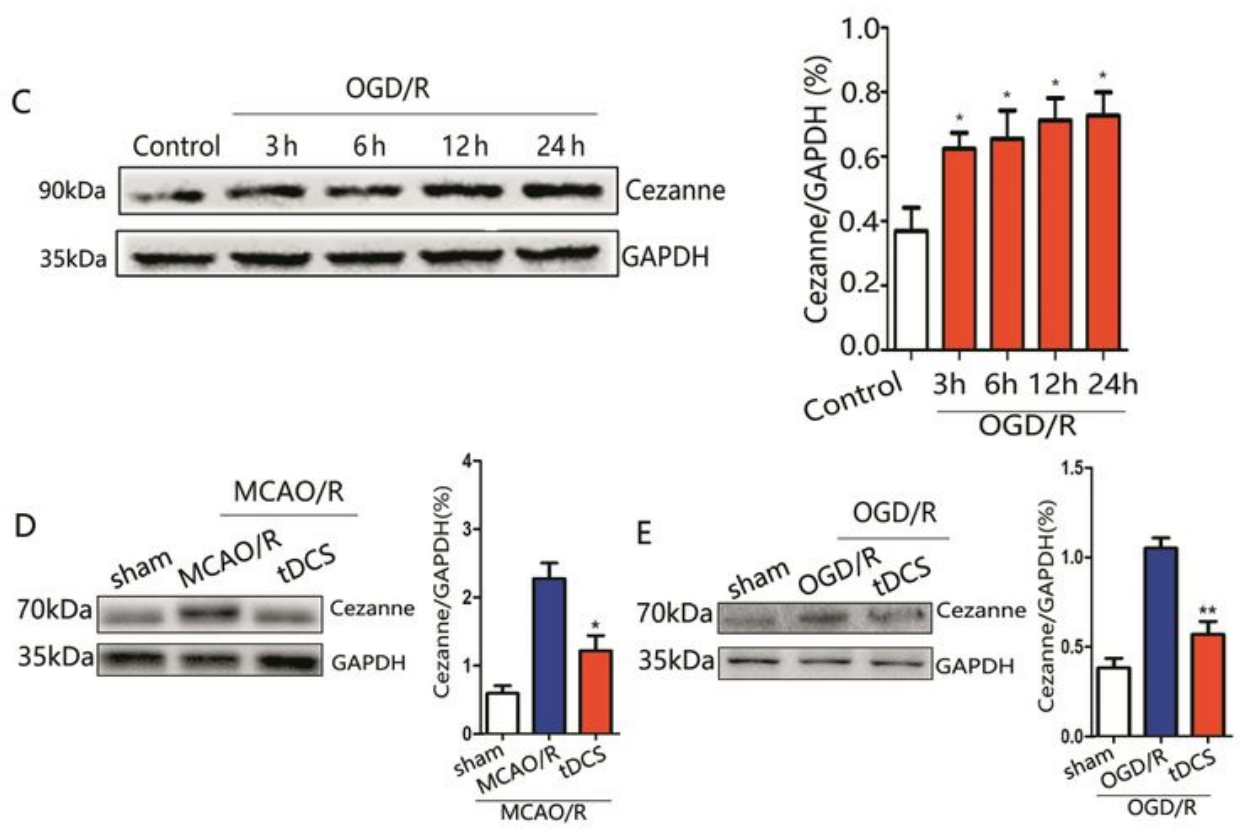

Figure 2

tDCS suppresses I/R-induced up-regulation of Cezanne in the ischemic neurons. (A) Double immunofluorescent staining of Cezanne and the neuron marker NeuN showed the changes in Cezanne 
expression in rat neurons treated with MCAO/R (90min/24h) compared to Control group. Control: rat normal brain tissues; MCAO/R: rat middle cerebral artery occlusion $90 \mathrm{~min} /$ reperfusion. (B) Western blotting analysis of the changes in Cezanne expression in MCAO 90min reperfusion at different time points of $3 \mathrm{~h}, 6 \mathrm{~h}, 12 \mathrm{~h}$ and $24 \mathrm{~h}$. Control: rat normal brain tissues;MCAO/R: rat middle cerebral artery occlusion $90 \mathrm{~min} /$ reperfusion. (C) Western blotting analysis of the changes in Cezanne expression in OGD 90min reperfusion at different time points of $3 \mathrm{~h}, 6 \mathrm{~h}, 12 \mathrm{~h}$ and $24 \mathrm{~h}$. Control: rat normal cortical neurons; OGD/R: the neurons were transferred to a deoxygenated, glucose-free extracellular solution for $90 \mathrm{~min} /$ restore oxygen and glucose. (D) In vivo, we established the MCAO/R ischemic stroke rat model, western blotting analysis of the changes in Cezanne expression in each group. Sham: rat with sham operation; MCAO/R: rat middle cerebral artery occlusion/reperfusion with nonstimulated tDCS; tDCS: rat middle cerebral artery occlusion/reperfusion with tDCS. (E) In vitro, we established the OGD/R injury cell model from the rat cortex primary neurons simulates brain I/R injury, western blotting analysis of the changes in Cezanne expression in each group. Sham: the neurons were transferred to a extracellular solution for $90 \mathrm{~min}$; OGD/R: the neurons were transferred to a deoxygenated, glucose-free extracellular solution for $90 \mathrm{~min} /$ restore oxygen and glucose with nonstimulated EF; tDCS: the neurons were transferred to a deoxygenated, glucose-free extracellular solution for $90 \mathrm{~min} /$ restore oxygen and glucose with EF. ( $n=6$ in each group, ${ }^{*}<0.05$ r $^{* \star} \mathrm{p}<0.01$ compared to Control/Sham, two-way ANOVA test, followed by Bonferroni post hoc test). 
A

LDH assay

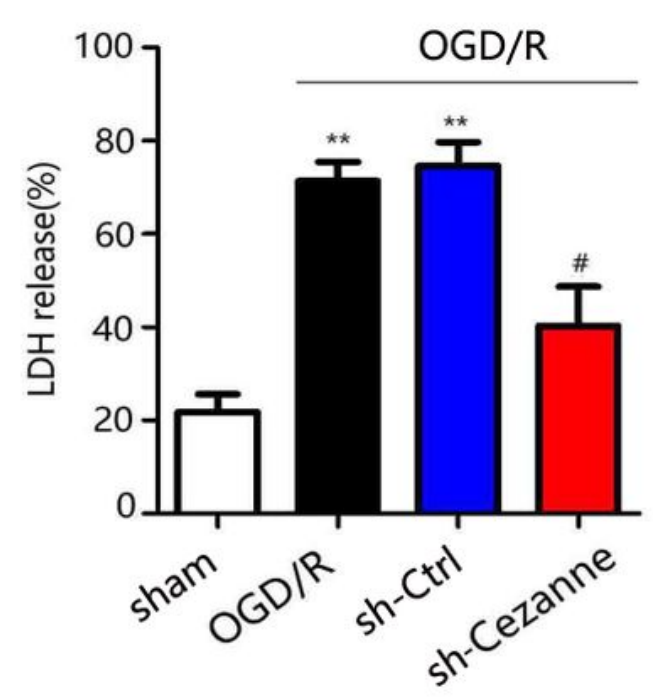

B

MTT assay

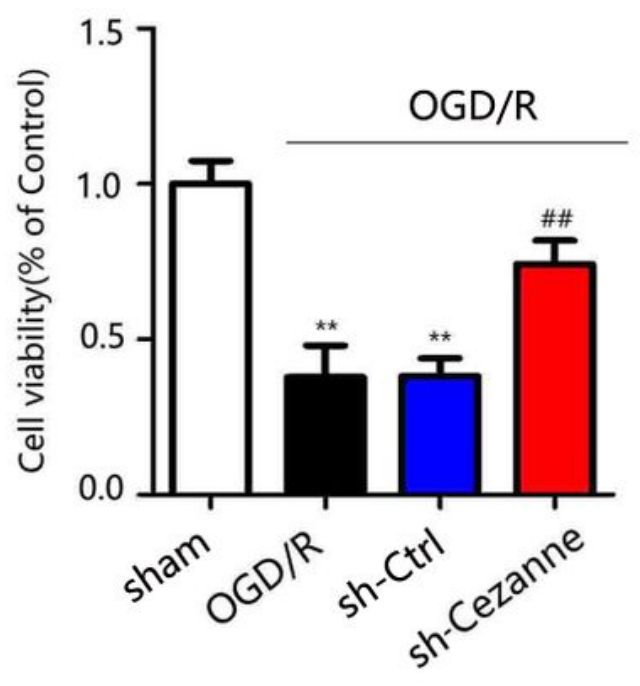

\section{Figure 3}

Suppressing Cezanne confers neuroprotection after rat cerebral ischemia injury. A) MTT assay showed that inhibiting Cezanne increased OGD-induced cortical neuronal survival rate. (B) LDH assay showed that inhibiting Cezanne reduced the release of OGD-induced cortical neuronal cytotoxic substance LDH. ( $n=6$ for each group; ${ }^{*} p<0.05$ or ${ }^{\star *} p<0.01$ compared to Sham, $\# p<0.05$ or \#\#p $<0.01$ compared to shCtrl, two-way ANOVA test, followed by Bonferroni post hoc test). 
A

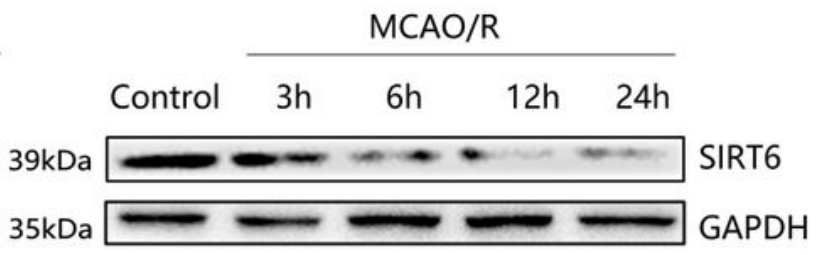

B
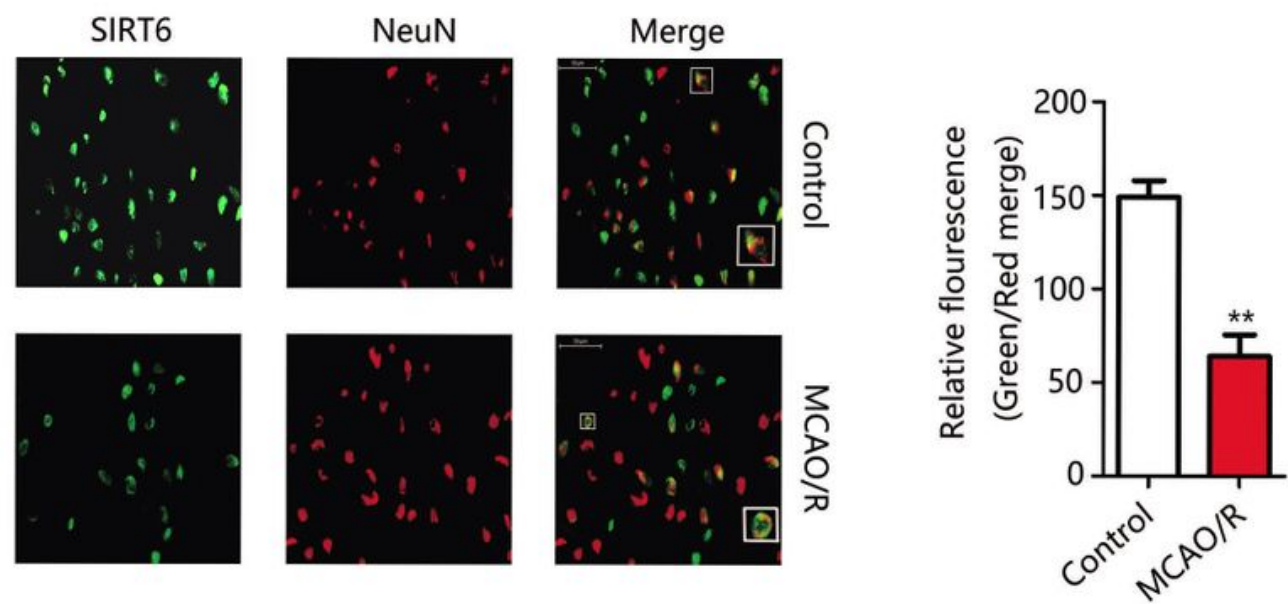

C

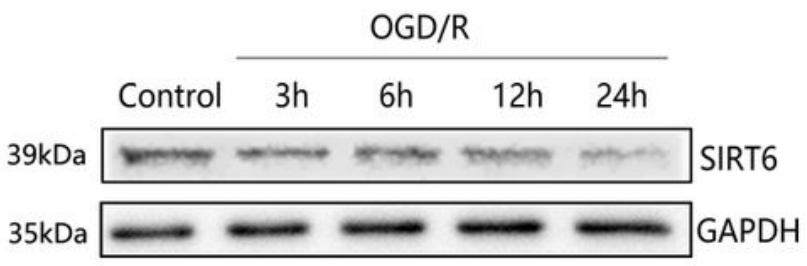

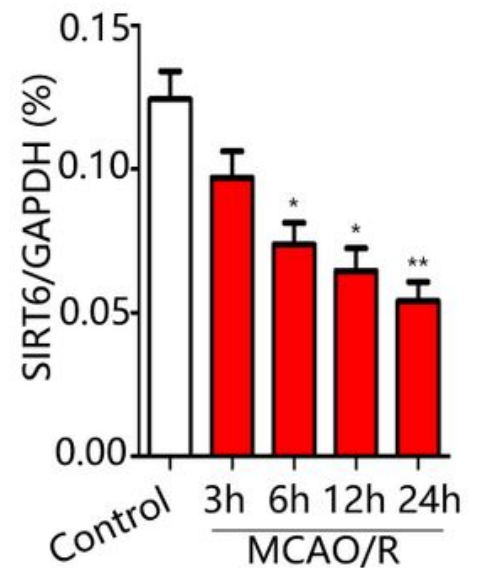

\section{Figure 4}

I/R-induced down-regulation of SIRT6 in the ischemic neurons. (A) Western blotting analysis of the changes in SIRT6 expression in MCAO 90min reperfusion at different time points of 3h,6h, $12 \mathrm{~h}$ and $24 \mathrm{~h}$. (B) Double immunofluorescent staining of SIRT6 and the neuron marker NeuN showed the changes in SIRT6 expression in rat neurons treated with MCAO/R (90min/24h) compared to Control group. Control: rat normal brain tissues; MCAO/R: rat middle cerebral artery occlusion 90 min/reperfusion. (C) Western 
blotting analysis of the changes in SIRT6 expression in OGD 90min reperfusion at different time points of $3 \mathrm{~h}, 6 \mathrm{~h}, 12 \mathrm{~h}$ and $24 \mathrm{~h}$. Control: rat normal cortical neurons; OGD/R: the neurons were transferred to a deoxygenated, glucose-free extracellular solution for $90 \mathrm{~min} /$ restore oxygen and glucose. $(n=6$ in each group, ${ }^{*} \mathrm{p}<0.05$ or ${ }^{* *} \mathrm{p}<0.01$ compared to Control, two-way ANOVA test, followed by Bonferroni post hoc test).
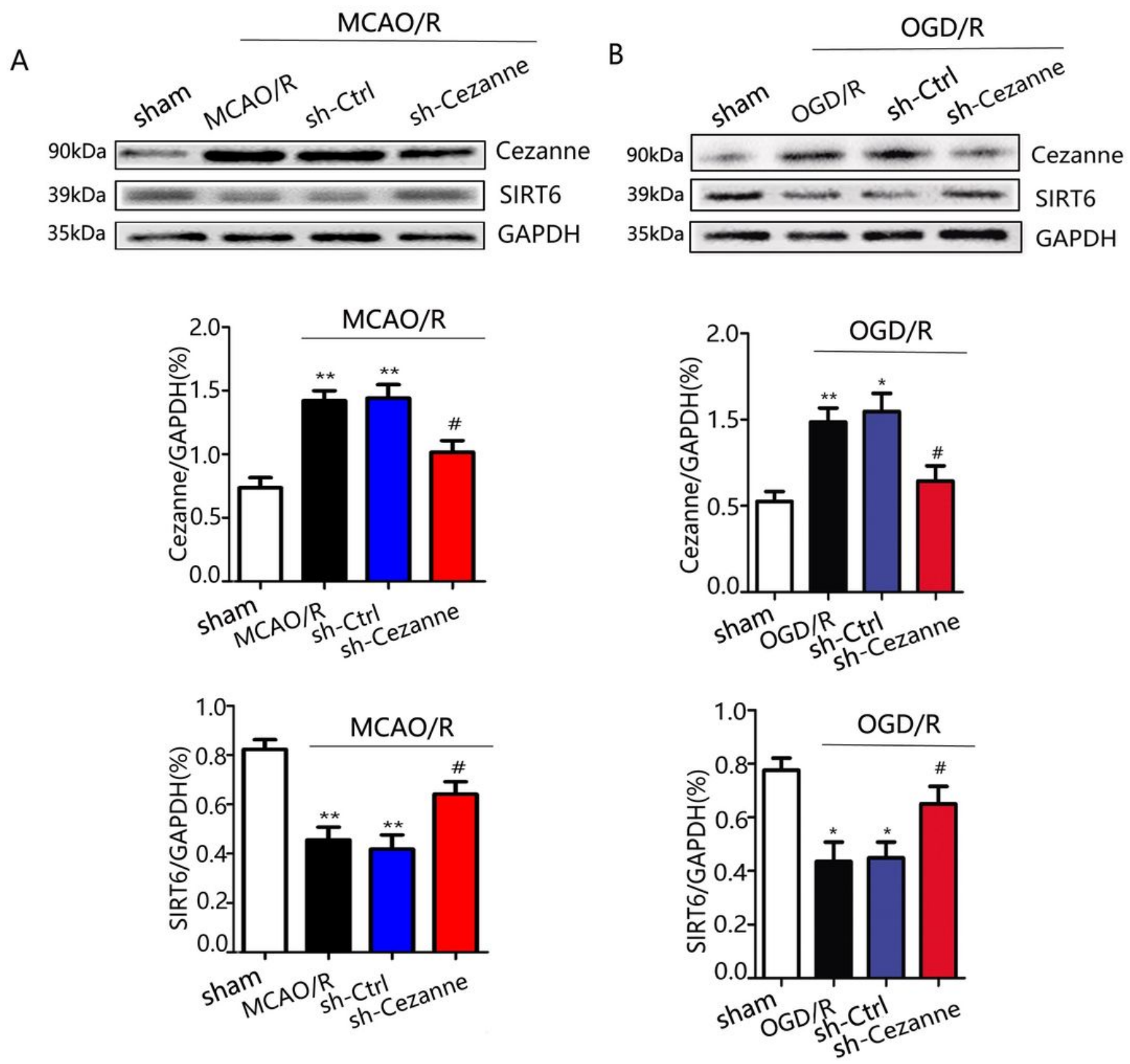

\section{Figure 5}

Suppressing Cezanne increases the level of SIRT6 after cerebral ischemia-reperfusion injury. (A) Western blotting analysis showed that the changes of SIRT6 and Cezanne protein level after the inhibition of 
Cezanne in rats treated with MCAO/R (90min/24h). Sham: rat with sham operation; MCAO/R: rat middle cerebral artery occlusion 90 min/reperfusion 24h;sh-Ctrl: control shRNA Lentiviral Particles was injected through i.c.v injection 1.5h following MCAO in rats before reperfusion 24h;sh-Cezanne: Cezanne shRNA Lentiviral Particles was injected through i.c.v injection 1.5h following MCAO in rats before reperfusion 24h. (B) In vitro, Western blotting assay showed that the level of Cezanne and SIRT6 protein in rat cortical neurons at 24 hours after OGD. Sham: the neurons were transferred to a extracellular solution for $90 \mathrm{~min}$; OGD/R: the neurons were transferred to a deoxygenated, glucose-free extracellular solution for 90 $\mathrm{min} /$ restore oxygen and glucose for $24 \mathrm{~h}$; sh-Ctrl: control shRNA Lentiviral Particles was transfected in neurons before constructing OGD/R injury cell model; sh-Cezanne: Cezanne shRNA Lentiviral Particles was transfected in neurons before constructing OGD/R injury cell model. ( $\mathrm{n}=6$ in each group. ${ }^{\star} \mathrm{p}<0.05$ or $\star \star p<0.01$ compared to Sham, \#p $<0.05$ or \#\#p <0.01 compared to sh-Ctrl, two-way ANOVA test, followed by Bonferroni post hoc test). 
A

MTT assay

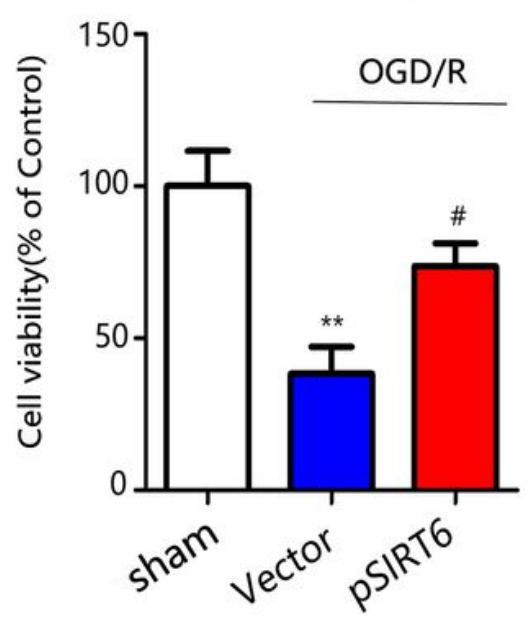

C

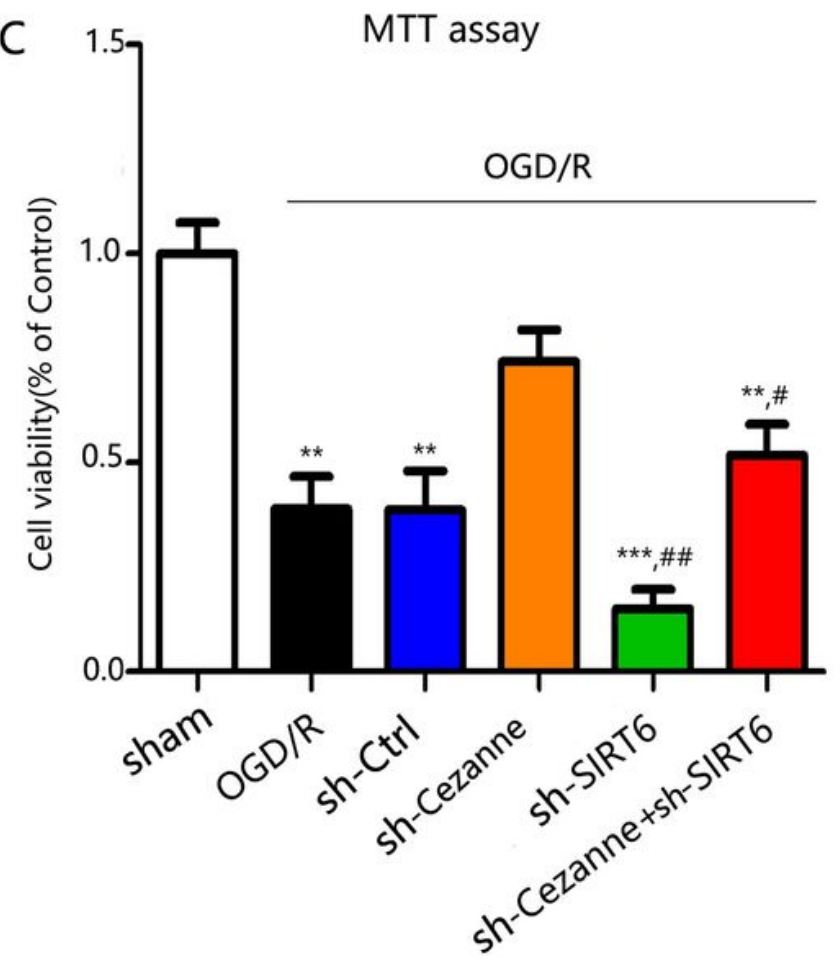

B

LDH assay

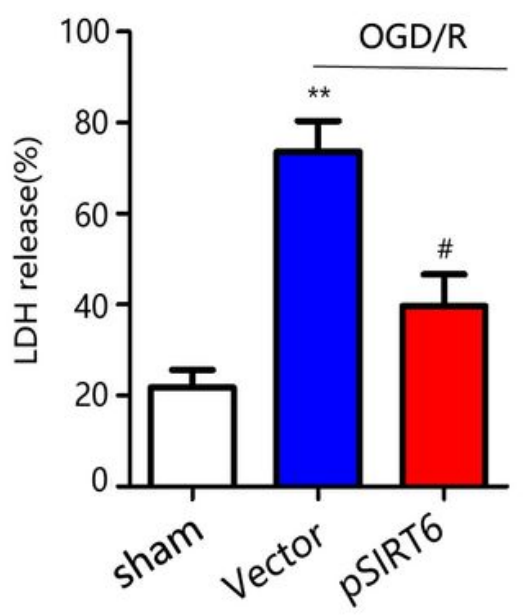

D

LDH assay

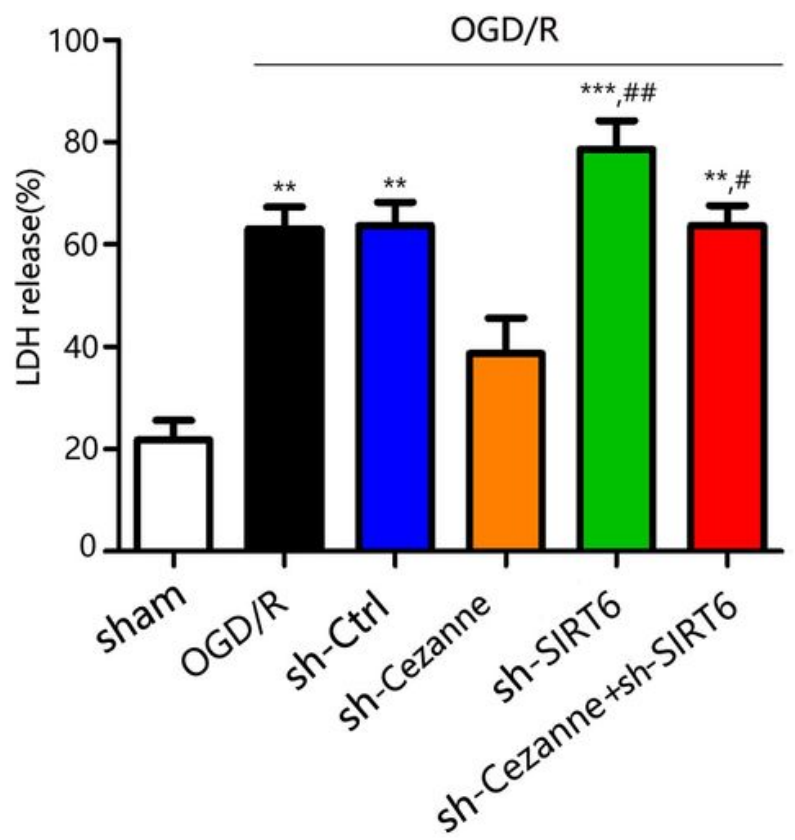

Figure 6

Overexpressing SIRT6 attenuates neuronal death and downregulating SIRT6 blocks Cezanne suppression-induced neuroprotection. (A) MTT assay showed that the changes of OGD-induced cortical neuronal survival rate in each group. (B) LDH assay showed that the changes of the release of OGDinduced cortical neuronal cytotoxic substance LDH in each group. (C) MTT assay showed that the changes of OGD-induced cortical neuronal survival rate in each group. (D) LDH assay showed that the changes of the release of OGD-induced cortical neuronal cytotoxic substance LDH in each group. $(n=6$ 
for each group; ${ }^{*} p<0.05,{ }^{* \star} p<0.01$ or ${ }^{* \star *} \mathrm{p}<0.001$ compared to Sham, \#p $<0.05$ compared to shCezanne/Vector, two-way ANOVA test, followed by Bonferroni post hoc test). sh-SIRT6: SIRT6 shRNA Lentiviral Particles were transfected in neurons before constructing OGD/R injury cell model. shCezanne+sh-SIRT6: SIRT6 shRNA Lentiviral Particles and Cezanne shRNA Lentiviral Particles were transfected in neurons before constructing OGD/R injury cell model. Vector: the negative control with pcDNA3.1 was transfected for $48 \mathrm{~h}$ in neurons before constructing OGD/R injury cell model; pSIRT6: the SIRT6 plasmid with pcDNA3.1was transfected for $48 \mathrm{~h}$ in neurons before constructing OGD/R injury cell model. 
A

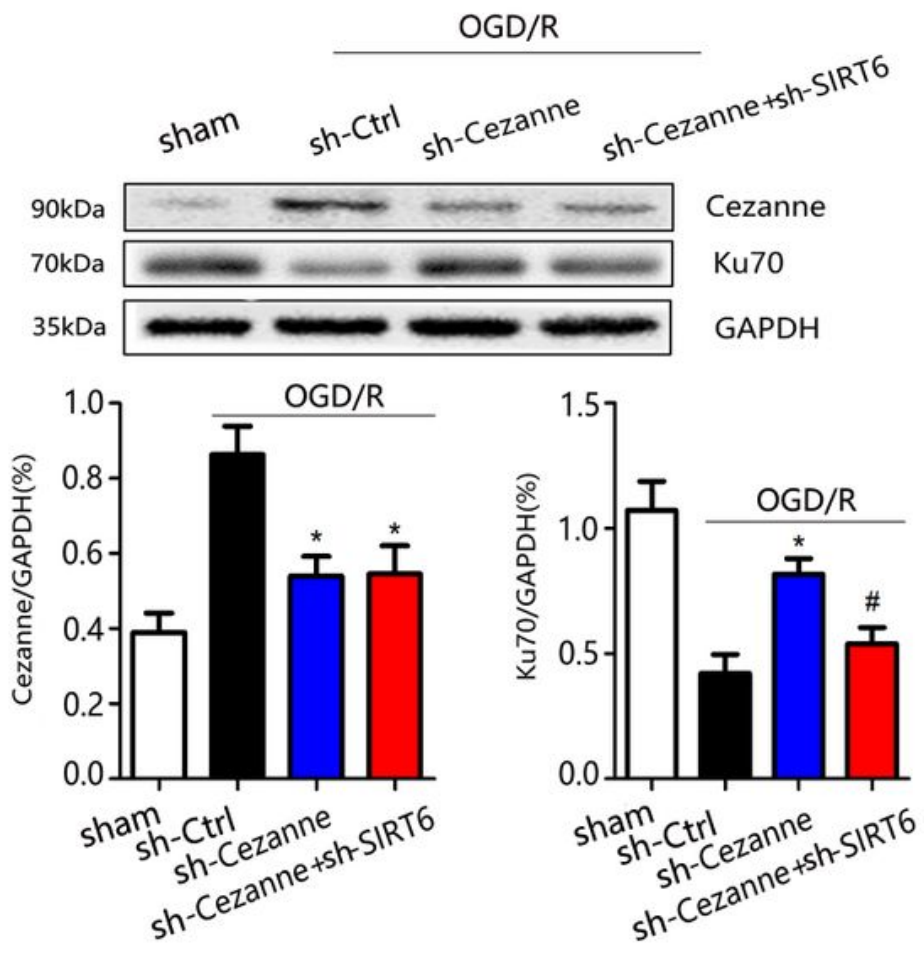

B
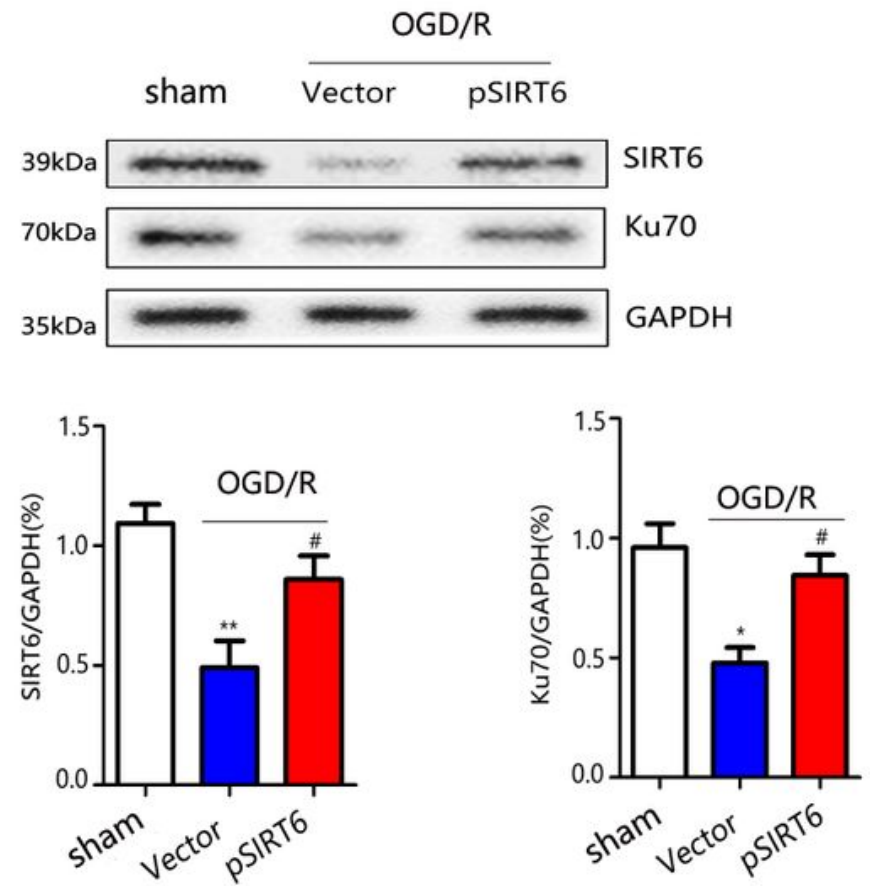

Figure 7

SIRT6 mediates the regulation of DNA DSB by Cezanne in OGD-insulted neurons. (A) SIRT6 shRNA Lentiviral Particles and/or Cezanne shRNA Lentiviral Particles were transfected to cortical neurons before the OGD stimulation. Western blotting assay showed that the Cezanne and Ku70 protein level in each group. (B) The SIRT6 plasmid with pcDNA3.1 was transfected for 48h in cortical neurons before the OGD stimulation. Western blotting assay showed that the SIRT6 and Ku70 protein level in each group. (n = 6 
for each group; ${ }^{\star} p<0.05$ or ${ }^{\star *} p<0.01$ compared to sh-Ctrl/Sham, \#p $<0.05$ compared to shCezanne/Vector, two-way ANOVA test, followed by Bonferroni post hoc test).

\section{Supplementary Files}

This is a list of supplementary files associated with this preprint. Click to download.

- Supplementary.docx 